\title{
La valoración del Practicum en la formación inicial: un estudio de casos internacional en el área de Geografía e Historia
}

\author{
José Ignacio MADALENA CALVO \\ Maria Francineila PINHEIRO DOS SANTOS \\ Liliana Angélica RODRÍGUEZ PIZZINATO \\ Xosé Manuel SOUTO GONZÁLEZ
}

Datos de contacto:

José Ignacio Madalena Calvo.

Profesor de Secundaria y co-director del curso de tutores del Máster de la UV. España.

jmada@telefonica.net

Maria Francineila Pinheiro dos Santos

Universidade Federal de Alagoas. Grupo de Pesquisa em Educação Geográfica - GPEG. Maceió, Brasil. francineilap@gmail.com

Liliana Angélica Rodríguez Pizzinato Universidad Distrital Francisco José de Caldas. Grupo de investigación Geopaideia. Bogotá, Colombia larodriguezp@udistrital.edu.co

Xosé Manuel Souto González Universitat de València. Proyecto Gea-Clío.Socials. València. España xose.manuel.souto@uv.es

Este trabajo forma parte del proyecto de investigación "Las representaciones sociales de los contenidos escolares en el desarrollo de las competencias docentes" (PGC2018-094491-B-C32), financiado por el Ministerio de Ciencia, Innovación y Universidades y cofinanciado con fondos FEDER de la UE

\section{RESUMEN}

El acceso a la función docente es asunto regulado por las instituciones administrativas, pero depende en gran medida de las competencias, actitudes y expectativas personales. Los estudios comparativos que mostramos revelan que las representaciones sociales que determinan un sentido práctico del saber escolar inciden en las opiniones de los actuales profesores de Secundaria y en los que aspiran a serlo. Este fenómeno subjetivo, que incide en las competencias profesionales, se interpreta cualitativamente con una metodología de casos, con la comparación de estos a partir de las encuestas y memorias del Practicum en tres universidades de Brasil, Colombia y España. El análisis sobre objetivos y competencias contrasta los deseos institucionales con las aplicaciones docentes, determinados por los discursos dominantes sobre la enseñanza escolar. Los resultados y conclusiones se relacionan con obstáculos y problemas identificados, cuando el alumnado en formación docente se acerca a los centros escolares. Más allá de un juicio de valor sobre la bondad/perversidad de las prácticas, se aboga por un estudio riguroso de las expectativas personales que condicionan la praxis profesional.

PALABRAS CLAVE: Practicum, representaciones sociales, estudios de caso, profesor de secundaria, praxis, investigación-Acción. 


\title{
The assessment of the Practicum in the initial training: an international case study in the area of Geography and History
}

\begin{abstract}
Access to teaching function is a matter regulated by administrative institutions, but depends to a large extent on competences, attitudes personal expectations. The comparative studies we show reveal that social representations that determine a practical sense of school knowledge affect the opinions of current and aspire to be high school teachers. This subjective phenomenon that affects professional competences is interpreted qualitatively with a methodology of cases in which the comparison of these from the surveys and reports of the Practicum in three universities in Brazil, Colombia and Spain. Analysis of objectives and competencies contrasts institutional desires with teaching applications, as determined by key discourses on school education. The results and conclusions relate to obstacles and problems identified when students in teacher training approach schools. The results and conclusions relate to obstacles and problems identified when students in teacher training approach schools. Beyond a value judgment on the goodness/perversity of internships, a rigorous study of personal expectations that condition professional practice is advocated.
\end{abstract}

KEYWORDS: Practicum, social representations, case studies, high school teacher, practice, action- research.

\section{Introducción}

La formación del profesorado de Secundaria en España ha adoptado la forma del criterio secuencial en la organización del Practicum. Primero se estudian los contenidos básicos de la disciplina, origen de los contenidos factuales, procedimentales y conceptuales. Después se estudian los contenidos propios del Master, que incluyen áreas genéricas (Psicologia, Pedagogía, Sociología) y específicas, propias, que desarrollará el futuro docente en Secundaria. Esta situación es diferente de la registrada en otros países, como Brasil y Colombia, ya que la formación como docentes se realiza en el Grado interrelacionando el saber disciplinar específico y el pedagógico. Por otra parte, los estudios sobre la futura formación inicial en España han incidido en potenciar esta parte de las prácticas profesionales (Fernández, 2001, Fernández \& Gurevich, 2018; Escudero, 2009; González-Sanmamed, 2015; Prats, Marín, \& Álvarez, 2019).

El diagnóstico institucional ${ }^{1}$ manifiesta la insatisfacción del alumnado con el escaso tiempo del Practicum del Máster de Profesorado

\footnotetext{
${ }^{1}$ Ver http://www.conferenciadecanoseducacion.es/wp-content/uploads/2018/02/documentoconferencia-decanos-desarrollo.pdf
} 
en Secundaria en España, además de señalar la escasa coordinación entre el profesorado, la poca conexión que ven entre el marco teórico y la percepción muy positiva del tiempo del Practicum (Souto, 2018; Solbes, Fernández, Domínguez, Cantó, \& Guisasola, 2018). Unas representaciones del Practicum, como opuesto a la teoria, que se refleja también en los casos de Brasil y Colombia (Santos, 2019; Rodríguez \& Souto, 2019), por lo que podemos conjeturar una representación social de las prácticas escolares como opuestas a la reflexión teórica. La construcción de este sentido práctico, que obedece a un sentido común, procedente del discurso del conocimiento vulgar, se puede cuestionar a la luz de los informes que realiza el alumnado de grado y postgrado y que son poco conocidos para otras personas que condicionan el funcionamiento del sistema escolar ${ }^{2}$.

Así comprobaremos que destacan la posibilidad de analizar las diferentes metodologías docentes, pues comparan sus objetivos teóricos estudiados en las licenciaturas y Máster con la observación de las aulas de Secundaria en sus visitas a los centros escolares. Las memorias apuntan a la necesidad de prolongar el tiempo del Practicum, tanto en el caso español (Universidad de Valencia) como en los casos analizados en los países de Brasil (Universidade Federal Alagoas) y Colombia (Universidad Distrital F.J. de Caldas), siguiendo una línea de trabajo que venimos desarrollando desde hace años (Rodríguez \& Souto, 2019; Santos \& Souto, 2018, Bertazzo, 2019).

En Brasil, a partir de la década del 2000, existe una nueva normativa legal (Parecer CNE/CP 009/2001 y la Resolución CNE/CP 001/2002), que explicitan los principios que guían la organización y estructuración de los cursos de Licenciatura, que indican las competencias y el conocimiento necesario para el desarrollo profesional, la organización institucional de la formación docente y las pautas para estructurar el currículo.

La relación entre universidades y escuelas en el Practicum es un camino que todavía se está construyendo, resultado de una relación compleja. Persisten dificultades en las responsabilidades y el compromiso que cada institución debe tener en la formación inicial del profesorado, pese a las mejoras introducidas en relación con investigaciones anteriores (Santos, 2012).

Las normativas legales se interpretan con el sentido práctico de cada persona para adoptar sus decisiones en el ámbito de la cotidianidad. Esto se refleja en la valoración del alumnado, que manifiesta explícitamente su agrado/desagrado con la estancia en los centros escolares durante el grado o el Máster. En especial, señalan la necesidad de consolidar los vínculos emocionales comunicativos con el

\footnotetext{
2 Seguimos las pautas metodológicas recogidas en los estudios de Domingos (2019), así como Domingos y Diniz (2019) que desarrollan las teorías de Moscovici (2011) y Jodelet (2005).
} 
alumnado y profesorado, tanto de la Universidad como de los centros escolares de enseñanza media o secundaria, según los países. Sobre todo, destaca el elemento emocional de su estancia, "los recuerdos que poseen como alumnos y la percepción superficial del ambiente educativo, condicionan la formación docente en los centros analizados" (Rodríguez \& Souto, 2019; 2), como lo corroboran estos tres ejemplos:

Desde mi punto de vista, las prácticas han sido absolutamente enriquecedoras ya que he entrado en contacto con el mundo de la docencia de pleno, he sufrido los contratiempos y retos que la práctica impone, así como he disfrutado de aquellos momentos de plenitud (A.D., $\left.\mathrm{V}-2020^{3}\right)$

Las relaciones no son cercanas, pues muchas investigaciones que se desarrollan en la Universidad en relación con la enseñanza no llegan a la escuela. Pero, cuando la Universidad necesita la escuela, como en el caso del Practicum, la escuela siempre está lista para ayudar (J.V.S., A.2012).

La importancia del maestro hoy es ser un mediador entre el conocimiento y los estudiantes, siempre pensando y re-pensado cada una de sus prácticas, para estar en el camino adecuado donde está la sociedad necesitada de transformaciones sociales y ciudadanos críticos. Sin embargo, el tiempo de la práctica es muy limitado... (A.D., B.-2019).

\section{Marco teórico para interpretar}

En la bibliografia existente sobre la formación del profesorado encontramos suficientes referencias sobre la preocupación desde posiciones teóricas (Coiduras, Gervais, \& Correa, 2009; Escudero, 2009; Perrenoud, 2004; Sánchez, 1996; Schön, 1992), en las que se ha sistematizado el registro de observación de la práctica escolar para entender la complejidad de la labor del profesorado de Secundaria, así como la problemática metodológica para entender los comportamientos de los sujetos docentes.

Las líneas de trabajo del grupo de enseñanza de la Geografia en Buenos Aires, nos parecen acertadas pues relacionan el estudio del sistema escolar, centrado en el análisis de la práctica escolar desde la acción de los alumnos universitarios en prácticas, con una temática de Ecología Regional y del Paisaje. El marco teórico elegido es el de la Investigación \& Acción, en la que colaboran docentes en ejercicio con el alumnado universitario, tomando como referencia los aportes disciplinares de la geografia (Fernández \& Gurevich, 2018).

\footnotetext{
${ }^{3}$ Las referencias a los documentos originales serán citadas de la siguiente manera: iniciales de la persona, Universidad Origen ( $V=$ Valencia, $B=$ Bogotá, $A=A$ lagoas) y año de recogida de la información. Los textos estaban escritos originalmente en portugués (Brasil), en castellano (Colombia) y en catalán y castellano en el caso español.
} 
Las tesis doctorales de Santos (2012) y Rodríguez (2019) inciden en la problemática del Practicum, como lugar de encuentro entre la reflexión teórica y el ejercicio de la práctica escolar. En este mismo sentido podemos citar el trabajo de Santos (2019) y Bertazzo (2019) que analizan comparativamente las experiencias de formación inicial en España y Brasil, utilizando como referencia la legislación de ambos países y las declaraciones del alumnado respecto a su valoración del período de estancia en los centros de secundaria.

Así pues, podemos concluir que el marco teórico referencial para las prácticas del Máster de Secundaria se encuentra en dos ámbitos de conocimiento. Por una parte, el normativo institucional y, por otra, el individual emocional; en un caso las decisiones de las autoridades administrativas son las que imponen unas reglas de juego y, en el otro, son las expectativas y deseos del alumnado quienes ofrecen una interpretación de los hechos sociales que se recogen en la comunicación educativa. Una sintesis que delimita un territorio epistémico (Rodríguez, 2019) donde confluyen las expectativas personales, los intereses gremiales, las decisiones institucionales y la conceptualización disciplinar.

Las reflexiones compartidas entre alumnos de práctica y docentes tutores y mentores, son importantes para comprender en sus teorias implícitas como asumen las experiencias educativas que están viviendo y desde las cuales actúan racionalmente (Clark \& Peterson, 1990). Estas teorías son apropiadas para dar regularidad y servir de estructura intelectual en los procesos de enseñanza y aprendizaje a los cuales se enfrenta (Rando \& Menges, 1991). Así, este conocimiento relevante para la acción suele ser tácito, ya que se desarrolla en y con la práctica y rara vez se pide que se explique. Por ello, identificar percepciones sobre el proceso de práctica, permitiría avanzar en la reflexión sobre acciones $\mathrm{y}$ valoraciones que hacen los sujetos en formación y ejercicio de la práctica pedagógica.

La legislación española respecto a la formación inicial en Secundaria se ha modificado en los años del siglo XXI. El Real Decreto de 8 de noviembre (1834/2008) define las condiciones de formación para el ejercicio de la docencia en la educación secundaria obligatoria, el bachillerato, la formación profesional y las enseñanzas de régimen especial ${ }^{4}$. Como se puede apreciar, las competencias administrativas complementan la regulación académica, no exenta de conflictos, para entender el funcionamiento del Master. El Practicum se valora con 10 créditos y un total máximo de 200 horas presenciales en los centros

\footnotetext{
${ }^{4}$ Las competencias educativas en España están descentralizadas en las Comunidades Autónomas, que regulan los requisitos administrativos de las Prácticas. En el caso de Valencia la Orden de 30 de septiembre de 2009, de la Conselleria de Educación, regula la convocatoria y el procedimiento para la selección de centros de prácticas.
} 
escolares.

En Colombia las normativas educativas proponen para las licenciaturas dar gran importancia a la pedagogía, como disciplina fundante con el Decreto 272 de 1998, lo que se acata en la acreditación previa de estos programas. El registro calificado para asegurar las condiciones de calidad de toda la oferta educativa del país es el Decreto 1295 de $2010^{5}$ y sus aplicaciones normativas que establecen mínimo 50 créditos presenciales (150 horas) dedicados a esta actividad en el plan de estudios. Esta normativa enfatiza que al docente en formación se le deben desarrollar habilidades para comprender las dinámicas escolares y del aula; además de asociarla con las situaciones educativas propias de la disciplina enseñada, la cual es pertinente para el problema tratado en el estudio de caso.

Por su parte, en Brasil, existe el Practicum, que es una actividad obligatoria y debe llevarse a cabo en las escuelas de Educación Básica, preferiblemente en las escuelas públicas. De acuerdo con la Resolución CNE/CP 002/2002, este debe sumar un total de 400 horas dedicadas en el área de formación y desempeño en educación básica, que debe ocurrir desde el comienzo de la segunda mitad del curso. Observamos así las diferencias en el número de horas de estancia en los centros escolares: Brasil 400 horas, España 200 y Colombia 150. Los debates sobre la organización del Practicum en los tres países, y en cada Universidad revela la necesidad de articular la teoría y la práctica, como manifiesta en el caso brasileño escogido 6 , que busca coordinar tutores universitarios y mentores de centros escolares en cuatro etapas ${ }^{7}$. Dichas normativas llegan a las universidades (en este caso Alagoas, Distrital F.J. Caldas y Valencia) y desde éstas se elaboran procedimientos de gestión, que busca la colaboración del profesorado de Secundaria en la formación del alumnado.

\section{Metodología del trabajo}

La metodología de trabajo tiene en cuenta las expectativas del alumnado que se quiere transformar en profesor/a de Secundaria. Así, se define el problema de trabajo en relación con los objetivos y competencias señaladas en las guías institucionales del Practicum, que se aplican con criterios de sentido práctico, determinados por el discurso dominante sobre la enseñanza escolar. Sólo en pocos casos se ha detectado a personas que son capaces de reflexionar sobre este proceso de

5 Con sus regulaciones administrativas para las licenciaturas (Decreto 2450 de 2015) y la reglamentación de la práctica pedagógica con la Resolución 2041 de 2016.

${ }^{6}$ Las normativas reguladoras son dos (Parecer CNE/CP 009/2001 y Parecer CNE/CP 009/2001)

${ }^{7}$ En el Practicum 1 y 2 se valoran las actividades de organización y estructura de la escuela, en el 3 y 4 se asumen los roles docentes. 
transformación de perspectiva de trabajo:

Si hago un trabajo de retrospectiva, reconozco que valoro mucho más el trabajo que esta persona realiza, como profesor en prácticas, más que como alumno suyo que había sido en su día (P.G., V.-2020) ${ }^{8}$

Como se ve, este alumno es consciente de su análisis sobre la tarea docente, en el momento que es capaz de contrastar su observación del período en prácticas con el recuerdo que tenía del momento en que acudía al mismo centro escolar como alumno de Secundaria. Algo más evidente en este segundo caso, que analiza los prejuicios de la observación de las clases de su mentor:

Cuando iba a empezar las prácticas y conozco al mentor, me surgió todo un conjunto de prejuicios. No entendía por qué continuaba dando clases magistrales con esquemas en la pizarra... ${ }^{9}$ (T.G., V.-2020)

Esta dificultad de valorar y categorizar las respuestas del alumnado en prácticas que oscilan entre la idealización de alumnos y la proyección de sus tareas docentes, o que proceden de una serie de prejuicios sobre lo que significa enseñar geografia e historia, se observa con detalle cuando valoran el trabajo del mentor/mentora y de sus metodologías docentes. Por eso mismo, los métodos que se van utilizar están determinados por el carácter cualitativo de las expresiones.

En un principio se aborda el estudio con diferentes encuestas para evaluar la observación de los docentes desde la posición del observador/a que son los alumnos del Postgrado y Licenciaturas en sus estancias o prácticas escolares. Con el caso de Valencia se realiza un muestreo de encuestas en cinco cursos sucesivos (entre 2015/2020), para valorar sus prácticas. Más tarde, se hizo el seguimiento de tres cursos escolares con 105 alumnos (2017 a 2020), con escala de Likert para valorar las actuaciones suyas en contraste con las de los mentores/as ${ }^{10}$. El estudio de los datos cuantitativos se sometió al análisis de pequeños grupos de trabajo y una posterior puesta en común por parte de los participantes en la misma encuesta. Los comentarios del alumnado del MAES del año $2020^{11}$ señalan, en efecto, que las concepciones sobre la enseñanza, producto del aprendizaje realizado en el Máster es determinante en la observación de la práctica escolar de sus mentores. Al contrastar tres preguntas sobre la metodología docente ha permitido verificar que el alumnado valora

\footnotetext{
${ }^{8}$ El texto original estaba redactado en catalán.

${ }^{9}$ El texto original también estaba redactado en catalán

10 Las puntuaciones máximas que se podrían alcanzar eran 108, 102 y 105 . De esta manera se podían ordenar las respuestas para facilitar el trabajo de pequeño grupo, con las personas que habían estado de estancia en los centros de Secundaria.

${ }^{11}$ Se organizaron ocho grupos de trabajo que analizaban las respuestas de sus encuestas (días 9 y 10 de marzo de 2020). Posteriormente, de forma individual (36 alumnos/as) interpretaron los resultados, que han quedado registrados en la plataforma virtual del curso.
} 
mejor la planificación que la puesta en práctica de la enseñanza de contenidos que son bloques de problemas sociales y ambientales. Es decir, existe una diferencia entre lo que se proyecta como contenido didáctico y lo que realmente se ejecutaba. Se verifica pues la incidencia del sentido práctico en la actuación docente, un obstáculo emocional que se debe combatir para tener algún tipo de éxito en la formación inicial.

Ello, obligó a revisar los criterios de acceso a las fuentes de conocimiento, siguiendo las pautas establecidas en otros estudios de metodología (Bisquerra, 2004; Coe, 2002; Moner \& Oller, 2014), así como las precisiones que se efectuaron en las Tesis doctorales mencionadas sobre los casos brasileiro y colombiano.

Los estudios de caso se refieren a la Universidad Federal de Alagoas (Brasil), la Distrital F.J. de Caldas (Colombia) y Valencia (España). Las observaciones del alumnado están condicionadas por su valoración del postgrado como un curso que se ha establecido como requisito administrativo para poder acceder a la función docente; una actitud que ya se ha comentado en otras ocasiones (Rivero \& Souto, 2019) y que se manifiesta en una radical separación entre la teoría y la práctica; categorizamos situaciones emocionales que se tratan de racionalizar desde el contexto de los casos estudiados.

Dado el carácter comparativo del estudio se ha buscado sólo mostrar los rasgos principales, que generan el sentido práctico avalado por un sentido común. Hemos comprobado que éste se ancla en los modelos tradicionales de enseñanza, que se objetiva como canon escolar.

\section{Fuentes}

Los casos estudiados corresponden a tres Universidades que tienen planes de estudios diferentes y con personas ejerciendo sus roles de alumnos y profesores, también distintas. En Valencia se analizan las memorias del Practicum de varios cursos escolares ${ }^{12}$. Una muestra intencional, pues se ha querido recoger afirmaciones de personas a las que se había acompañado en sus estancias en los centros escolares ${ }^{13} \mathrm{y}$ así poder entender mejor sus palabras. Además, se registra la intervención de un total de 44 profesores en tres cursos sucesivos.

Los informes anteriores al año 2017 se conservan en papel en los archivos del Máster, mientras que a partir de ese curso existe una selección de los mismos en la web del aula virtual ${ }^{14}$. Hemos traducido

\footnotetext{
12 La tabla 1 indica el número de memorias analizadas. Los años aparecen reflejados junto a los acrónimos de las personas y universidad, como se ha señalado en nota 3.

${ }_{13}^{13}$ Un antecedente de este modelo de trabajo lo podemos ver en Císcar et al. (2011)

14 Practicum 2017-18: https://aulavirtual.uv.es/mod/folder/view.php?id=52272; Practicum 201819: https://aulavirtual.uv.es/mod/folder/view.php?id=52249 y Practicum de 2019-20:
} 
los textos en catalán al castellano para facilitar la lectura ${ }^{15}$, tal como hemos hecho con las memorias y entrevistas de Brasil, que están escritas originalmente en portugués ${ }^{16}$. Las memorias de Colombia hacen parte de la información recolectada en la tesis doctoral de Rodríguez (201917).

En el caso de la Universidad Federal de Alagoas se analiza el grado de Geografia Licenciatura compuesto por grupos de 40 estudiantes cada semestre, con un total de 80 alumnos al año; si bien sólo la mitad concluye este tipo de estudios. La formación se corresponde a la profesión que se puede realizar en educación básica18. Se seleccionaron un total de nueve Memorias entre las sesenta y cuatro que se han analizado ${ }^{19}$. Se tiene evidencia de entrevistas y opiniones de los profesores mentores del Practicum 1, 2, 3 y 4 de los centros escolares presentes en la tesis de Santos (2012), así como otras entrevistas con cuatro profesores mentores del Practicum de cuatro centros escolares y tres profesores tutores del Practicum, todas en 2019.

En el caso de la Universidad Distrital F.J. Caldas los alumnos del estudio cursan la última parte de la carrera en un número aproximado 120 por año, con actividades referidas a la caracterización del contexto escolar o comunitario donde realizarán la práctica en VIII semestre. Se cuenta con un total de 66 observaciones de alumnos/as.

Tabla 1.

Memorias analizadas

\begin{tabular}{|l|c|c|c|}
\hline Universidades & F.J. Caldas Bogotá & Federal de Alagoas & València \\
\hline $\begin{array}{l}\text { Alumnos } \\
\text { Informes }\end{array}$ & 62 & 64 & 66 \\
\hline $\begin{array}{l}\text { Profesores } \\
\text { opiniones }\end{array}$ & 4 & 7 & 54 \\
\hline
\end{tabular}

https://aulavirtual.uv.es/mod/assign/view.php?id=195543

${ }^{15}$ Sólo hemos conservado alguna frase en su idioma original cuando hemos considerado que el giro utilizado era difícil de transcribir sin perder el sentido original

${ }^{16}$ Las Memorias de Prácticas del alumnado del curso de Geografía Licenciatura se encuentran depositadas en el Departamento de Geografía, Desenvolvimento e Meio Ambiente. Universidade Federal de Alagoas/Brasil.

${ }^{17}$ La información fue procesada en el software Qualitative Data Analysis ATLAS-Ti.8, con licencia de funcionamiento Versión 8.2.1 (550) Mac/Windows. Estudiante Licencia de usuario individual. (2018-2020). Alemania.

${ }^{18}$ Hemos analizado las memorias del Practicum 1, 2, 3 y 4 presentes en la tesis de Santos (2012) y las memorias de cuatro clases, siendo dos del primer semestre y dos del segundo semestre, todas del año de 2019

19 Para el primer semestre hemos analizado ocho memorias para cada Practicum, un total de treinta y dos memorias del Practicum 1, 2, 3 y 4 . Para el segundo semestre hemos analizado la misma cantidad. 
La consideración de las creencias docentes sobre sus supuestos pedagógicos, psicológicos, habilidades de planificación, actuación y evaluación o esquemas de procesamiento de información, utilizados para adecuarlos con las estrategias de acción es un proceso de toma de decisiones constante. En los momentos de la actividad profesional, el de programación o preactivo del proceso de enseñanza y el activo que afecta la actuación y acción en el aula (Hernández y Sancho (1996), pueden contribuir a una mejor concepción del enseñante como profesional. Además, el profesorado realiza una percepción selectiva del medio escolar, lo que significa que su actuación no es totalmente objetiva ni neutral, sino que se apoya en sus propias ideas, creencias y expectativas (Pérez, 1987). Esto puede servir como referente para plantear una formación inicial, para ser conscientes de las concepciones que fundamentan la práctica.

Para buscar un diálogo entre el pensamiento del profesorado en activo y el futuro docente (en su momento alumnado en formación) se tienen en cuenta las opiniones manifestadas por los profesores/as que han participado en los Seminarios de Formación organizados por el Máster de Profesor en Secundaria de la Universidad de Valencia ${ }^{20}$.

El formato de este seminario ha combinado sesiones presenciales y actividades desarrolladas a distancia por medio de la plataforma Moodle; las sesiones presenciales tenian como objetivo conectar la práctica de tutorización con la reflexión sobre la formación de los docentes. En cuanto a las sesiones no presenciales, se han centrado en la lectura reflexiva de artículos sobre diversas preguntas: ¿cómo se accede a la profesión docente?, ¿cuáles son las competencias profesionales de los docentes?, ¿cómo se enseña y cómo se evalúa la formación de los tutorados del Practicum del Máster?, que permitían profundizar en la formación de los propios docentes mentores.

\section{Recolección e interpretación de datos}

Para recolectar los datos se seleccionaron dos categorías conceptuales principales: la valoración del alumnado de sus mentores o profesoras que les acompañan en su estancia en los centros de Secundaria y su propia autoevaluación respecto al trabajo como futura docente. Estas categorías conceptuales remiten a una investigación cualitativa, determinada por el comportamiento y emociones que despiertan en el alumnado el trabajo docente de otras personas y del suyo en el futuro. Un fenómeno que es muy difícil de objetivar, pero que determina claramente las rutinas, tradiciones y guiones profesionales del profesorado.

\footnotetext{
${ }^{20}$ Los seminarios se realizaron en los cursos 2017/18, 2018/19 y 2019/20, con un total de 18,21 y 15 participantes respectivamente.
} 
Tabla 2.

Declaraciones alumnado

\section{a) La opinión del alumnado del Practicum sobre la relación con los profesores mentores.}

YC., B.-2017: El $\quad$ L.D.R.N., A.-2019: La mayoría docente siempre debe estar dispuesto a escuchar las dudas e inquietudes de sus alumnos, reconociendo que como persona no puede conocerlo todo, y de la misma forma no todo lo que sabe es lo más correcto, pero que mientras intente cultivar, la reflexión, el cuestionamiento y la investigación en el corazón de sus alumnos estará cumpliendo su labor.

de los profesores mentores que me acompañaron en las escuelas me dieron la oportunidad de conciliar la teoría y la práctica. Estuvieron muy atentos, me hablaron sobre las clases antes y después de salir, me aconsejaron sobre cómo hablar con los estudiantes de la clase. Sin embargo, creo que (...) algunos no están preparados para recibir estudiantes del Practicum y terminan limitando nuestra oportunidad de aprender en la práctica. Estaba muy angustiada cuando un profesor mentor no me permitía usar juegos educativos en clase y exigía que la clase fuera teórica, de acuerdo con su planificación. Este profesor mentor no me permitió usar la dinámica en la clase.

KA., B.-2012: Lo que verdaderamente ayudó en $\mathrm{mi}$ formación para $\mathrm{mi}$ labor docente fue la planeación para la unidad didáctica, ese primer acercamiento a lo que sería organizar mis clases, como las desarrollaria, de que hablaria, para mí fue algo muy bueno, el trabajar en equipo durante el semestre fue algo duro y complicado para mí.
R.A.S., A.-2019: Los profesores mentores que me acompañaron en las escuelas fueron buenos y serviciales. (...). Noté que los maestros se enfrentan a muchas dificultades en las escuelas, no tienen recursos ni materiales, por lo que terminan optando por una enseñanza muy tradicional, con poca o casi ninguna innovación. El problema es que manejan la forma en que imparten clases en el interés de los alumnos y mucho menos en la criticidad de ellos. En cierto modo, me sentí frustrado por la falta de incentivos de los maestros para que los alumnos aprendan.
A.D., V.-2020: Mi experiencia con la mentora ha sido muy enriquecedora, puesto que desde el primer momento depositó en mi plena confianza y me dio total libertad para intervenir en las clases y prepararme para impartirlas...la tarea de observación que he ejercitado con ella no se ha limitado a aspectos exclusivamente del aula, sino que el proceso de formación (...) me ha ayudado a comprender todas las dinámicas que se desarrollan dentro de un centro de enseñanza, desde la gestión del aula, a la atención de padres, las normativas.

I.G., V.-2020: En cuanto a la relación con mi mentora me satisface decir que ha sido excelente. Siendo una convivencia intensa durante tres meses no he le visto un mal gesto; ella y el claustro al completo nos han acogido a los alumnos/as de prácticas con los brazos abiertos; nos han facilitado toda la documentación que pediamos y nos ofrecian documentos adicionales, etc. 
Las memorias analizadas nos ponen de relieve el sentido emocional de las observaciones realizadas por el alumnado: "estuvieron muy atentos", "depositó en mí plena confianza", "el corazón de los alumnos" respecto a la figura de la mentora/mentor. Pero al mismo tiempo indican las dificultades de coordinación entre tutores de Universidad y mentores de escuelas: "Es dificil organizarse para trabajar con unas directrices que te dan en la universidad y otras las que dan los profesores en las escuelas" (D.C., B.-2019).

La sintesis de estos registros resalta un obstáculo en la observación de los docentes: "algunos profesores no eran comunicativos, estaban cansados, desmotivados..." Ello ocasiona problemas como: prohibiciones de juegos educativos y dinámicas en clase y exigencias para las clases teóricas de acuerdo con su planificación.

Como contraste podemos observar la opinión del profesorado en sus roles de tutores y mentores respecto a las competencias para acceder a la función docente. En el caso de Valencia se realizaron al menos 4 intervenciones de cada profesor, si bien varios de ellos intervinieron más, replicando a otras entradas en el foro. Por tanto, el número de entradas superaría las 200, a las que habría que sumar las de los profesores tutores de la Universidad 21 .

Las opiniones recogidas, así como los debates presenciales en los seminarios citados, han puesto de relieve opiniones coincidentes con las registradas en los casos de Colombia y Brasil. Así se hace hincapié en las actitudes del alumnado respecto a la praxis (en especial, la metodología), a la observación de las clases y a la capacidad de los/las mentores para dirigir el trabajo del futuro docente desde su experiencia. Al mismo tiempo, hay coincidencias con lo expresado por el alumnado respecto al tiempo de permanencia en los centros escolares.

Tal como sucedía con la interpretación de la labor de la mentora o tutor, lo que se aprecia es un conjunto de emociones, expectativas e intereses que se alejan de una cuantificación de indicadores y se acercan al mundo de las representaciones sociales del profesorado.

\footnotetext{
${ }^{21}$ Las referencias a las URL de estos cursos son las siguientes: Curso 2019-20 "Tutorització de l'alumnat del màster en professorat d'educació secundària". http://cefire.edu.gva.es/course/view.php?id=20596 Curso 2018-19: "Tutorització de l'alumnat del màster en professorat d'educació secundària" http://cefire.edu.gva.es/course/view.php?id=18565 y Curso 2017-18. "Tutorització de l'alumnat procedent del màster en professorat d'educació secundària" http://cefire.edu.gva.es/course/view.php?ld=15967
} 
Tabla 3.

Declaraciones profesorado

\section{b) La opinión de los profesores tutores sobre las competencias}

de los alumnos para impartir las clases.

\begin{tabular}{|c|c|c|}
\hline $\begin{array}{l}\text { S.V., B.-2019: Se } \\
\text { recomienda incorporar } \\
\text { una estrategia inicial } \\
\text { de exploración de } \\
\text { ideas previas, seguida } \\
\text { de la experimentación, } \\
\text { la cual se puede } \\
\text { complementar con la } \\
\text { lectura del libro que } \\
\text { aporta elementos de } \\
\text { comprensión del tema. }\end{array}$ & $\begin{array}{l}\text { R.L.A., A.-2019: Me doy } \\
\text { cuenta de que los } \\
\text { estudiantes necesitan } \\
\text { mejorar la articulación } \\
\text { entre el conocimiento } \\
\text { teórico y la práctica en } \\
\text { el Practicum. A algunos } \\
\text { estudiantes les resulta } \\
\text { muy dificil llevar a cabo } \\
\text { el Practicum, tanto } \\
\text { desde el punto de vista } \\
\text { teórico como } \\
\text { metodológico, así como } \\
\text { las emociones para } \\
\text { lidiar con el aula. }\end{array}$ & $\begin{array}{l}\text { M.C.R., V.-2020: } \\
\text { Yo pienso que es } \\
\text { necesario que los } \\
\text { alumnos del Master } \\
\text { pasen más tiempo en } \\
\text { aulas reales con } \\
\text { alumnos reales siendo } \\
\text { uno de los objetivos } \\
\text { esenciales... (para } \\
\text { pensar) si esa profesión } \\
\text { es la que elige ejercer. }\end{array}$ \\
\hline $\begin{array}{l}\text { H.A., B.-2019: Se } \\
\text { espera Licenciados } \\
\text { sumamente objetivos } \\
\text { que fundamenten su } \\
\text { quehacer desde la } \\
\text { calidad formativa } \\
\text { recibida que los hace } \\
\text { idóneos para un } \\
\text { desempeño calificado } \\
\text { que les permita hacer } \\
\text { de su quehacer un } \\
\text { proceso investigativo. }\end{array}$ & $\begin{array}{l}\text { G.R.S., A.-2019: - Creo } \\
\text { que algunos estudiantes } \\
\text { del Practicum no tienen } \\
\text { interés, no preparan las } \\
\text { clases como deberian y } \\
\text { cuando participan en } \\
\text { clases, experimentan } \\
\text { algunas dificultades } \\
\text { tanto en el conocimiento } \\
\text { geográfico como en la } \\
\text { relación con los } \\
\text { estudiantes de la } \\
\text { escuela. Por lo tanto, } \\
\text { hay clases } \\
\text { insignificantes para el } \\
\text { proceso de enseñanza- } \\
\text { aprendizaje. }\end{array}$ & 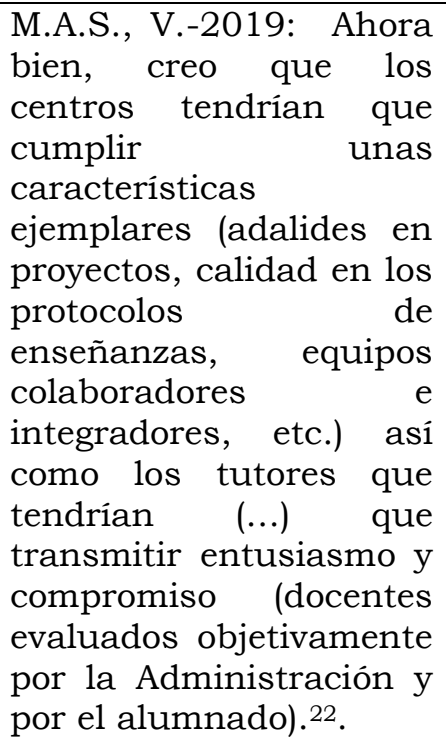 \\
\hline
\end{tabular}

Por su parte, el alumnado registraba en sus memorias más descripciones de emociones y sensaciones en el desarrollo de su actividad docente, pero también indican sus decisiones para transformar las dinámicas de las clases. Ello permitía observar las coincidencias de los tres casos.

\footnotetext{
22 Texto original en catalán.
} 
Tabla 4.

Prospectiva alumnado

\section{C) Descripción de su propio yo en el futuro}

K.A., B.-2019: A veces me da un poco de miedo no saber cómo manejar situaciones $\mathrm{y}$ levantarme un día y ver que soy esa profesora que va lee un libro, o se para frente al tablero y habla y habla pero que en realidad no está haciendo nada con su vida ni la de sus estudiantes, por esto es imprescindible en lo que a mi respecta estar cambiando reinventándome, ver que existen más posibilidades que andar quejándose, que en realidad si se puede ser feliz con lo que se hace (Y.T. B-17, 2017).

J.B., B.-2019-Es de vital importancia como maestros en formación plantearnos estas inquietudes para así proyectar nuestra futura labor como educadores y transformadores de realidades, ya que como maestros tenemos la capacidad de hacer que las realidades cambien en los sujetos con nuestra actuación, claro está que esto sucede si no lo proponemos con toda la seriedad y responsabilidad del caso.
L.D.R.N., A.-2019: El Practicum tuve contacto con los alumnos de la escuela, con la preparación de las clases y de las pruebas. Aprendí mucho en la escuela y espero que cuando sea maestra ayude a mis alumnos a ser sujetos creativos y dinámicos.

R.A.S., A.-2019: En el Practicum, observo muchos problemas en la escuela, tanto en términos de infraestructura y falta de materiales, como de los mentores muy tradicionales y a quienes no les importa mucho el aprendizaje de los alumnos. Tengo la intención de ser un maestro que traiga material nuevo a las clases, con tecnología para ayudar a mis alumnos a comprender la importancia de la geografia.
J.R., V.-2017: La experiencia como docente en prácticas en las aulas me ha resultado muy gratificante. Al igual que la mayoría de compañeros en el Máster de Secundaria, considero que ha sido el período más enriquecedor de este curso en relación con los procesos de enseñanza y aprendizaje de las Ciencias Sociales, pero también respecto a los problemas personales, sociales y profesionales que alumnos y profesores sufren en la escuela y en el entorno de ésta.

LG., V.-2020:

Asimismo, en estos grupos de $1^{\circ}$ pude observar cómo trabajaron a partir del aprendizaje

cooperativo. (...) los alumnos estudiaron la prehistoria cambiando la organización del aula. Así pues, si con anterioridad se sentaban en parejas, ahora formarian grupos de entre 4 y 5 personas elegidos $y$ distribuidos

previamente por el profesor en función de las necesidades 


\section{Hechos y conclusiones para continuar}

La comparación de situaciones analizadas nos ha permitido avanzar en algunas coincidencias que faciliten la interpretación de los obstáculos y problemas que surgen en el momento de acercase un alumnado en formación docente a los centros escolares. Se han establecido categorias conceptuales para avanzar en la interpretación desde diferentes marcos conceptuales.

- Las relaciones de comunicación alumnado/tutor/mentor

- Las referencias teóricas a la profesión docente para orientar la observación de aula, de centro y del contexto social y profesional

En primer lugar, analizamos las observaciones realizadas desde un juicio emocional, claramente influido por sus recuerdos, expectativas y conocimientos adquiridos en las clases teóricas del postgrado o de licenciatura, según sea el lugar de origen de las opiniones recogidas.

\section{El juicio del alumnado desde las representaciones sociales del profesorado}

Se comprueba que las expresiones muestran una prioridad en la comunicación personal más que lo que pueda generar de conocimiento profesional. Al igual que ha sucedido en los estudios de la enseñanza de la Historia (Martínez, 2014) la figura del profesor se analiza desde su comportamiento personal en una clase, los denominados guiones de actividad, que marcan las rutinas, en las que las emociones son clave.

Tres de las cuatro expresiones recogidas muestran la satisfacción con sus mentores (buenos, atentos, comprometidos) y uno cuarta desacredita su trabajo por oponerse al criterio de la innovación, ya que existe la sospecha de que no sirve para incorporar un conocimiento alternativo (según la observación realizada). Ello supone la elaboración de juicios de valor centrados en cuestiones emocionales, que después repercutirán en la manera de concebir la enseñanza, pues estas opiniones se acaban transformando en hábitos y rutinas.

Los profesores mentores que me acompañaron en las escuelas fueron buenos y serviciales...(R.A.S., A.-2019)

En cuanto a la relación con mi mentora me satisface decir que ha sido excelente....(I.G., V-2020)

En el Practicum 3 y 4 los profesores mentores fueron comprometidos y atentos...(A.E.O.S., A.-2019).

Tiene una visión negativa de las actividades de clase innovadoras... como pérdida de tiempo o recompensa de comportamiento, pero no como forma de incorporar conocimiento (C.B., V.-202023)

\footnotetext{
${ }^{23}$ Texto original en catalán.
} 


\section{Relaciones con mentores: acogimiento al alumnado en Prácticas}

La sensación que el alumnado tiene sobre sus mentores es otra categoría conceptual que se puede analizar desde las emociones y comportamientos. Los ejemplos seleccionados son contrastados y se centran en el control o en la libertad que les conceden para realizar sus tareas. Es decir, cifran el éxito de su entrada en el ejercicio docente en la capacidad de libre albedrio para llevar a cabo un programa de enseñanza, pues el alumnado suele llegar a los centros con prejuicios sobre cuál es el significado de enseñar bien. El calificativo moral es coherente con el planteamiento del alumnado.

Creo que algunos profesores mentores están entrenados para realizar el ejercicio en las clases, pero algunos no están preparados para recibir estudiantes del Practicum y terminan limitando nuestra oportunidad de aprender en la práctica (L.D.R.N., A.- 2019)

Me dio total libertad para intervenir en las clases y prepararme para impartirlas. Diría que la tarea de observación que he ejercitado con ella no se ha limitado a aspectos exclusivamente del aula, sino que el proceso de formación se ha consolidado a lo largo de todo el periodo de prácticas y me ha ayudado a comprender todas las dinámicas que se desarrollan dentro de un centro de enseñanza (A.D., V.-2020)

Hay una fuerte desarticulación entre lo que nos enseña el tutor en la práctica en la universidad y lo que nos pide el mentor en el colegio. Parecen dos realidades diferentes e irreconciliables (D.G., B.-2020).

\section{Las referencias teóricas a la profesión docente}

Para ello hemos buscado las frases de los informes y de las entrevistas en las cuales se manifestaba la valoración del trabajo docente. Se puede comprobar que existe un juicio de valor respecto a algún paradigma o marco teórico de referencia (tradicional/innovador), que seguramente procede de las clases teóricas de sus cursos universitarios. (Esteve, 2006; Escudero, 2009). La representación social se justifica desde los modelos o paradigmas del profesorado (tradicional, técnico, espontaneísta, crítico). Es decir, se juzga el trabajo del docente/mentora desde planteamientos globales, como se puede verificar en los conceptos utilizados o en el valor absoluto que se le concede al libro de texto, lo que supone un avance sobre las categorías anteriores.

Valentí es un profesor que se esfuerza en ser innovador... (P.G., V.2020)

Me doy cuenta de que un punto débil es que algunos profesores mentores están demasiado apegados al libro de texto y no usan otros recursos. (J.E.B.S., A.-2019) 
El libro se gastaba como una guía sobre los contenidos que iban dando y el alumnado lo utilizaba durante les clases...(E.S., V.-202024)

Ser un docente investigador es lo que dice nuestro perfil profesional, pero en la escuela tradicional esto es dificil de hacer y el equipo de área de ciencias sociales piensa que es una idea romántica y que se ve desde la universidad alejada de la realidad. (K.A., B.-2019)

Es de suma importancia tener un conocimiento amplio acerca de lo que se va a enseñar pero esto va acompañado de la actitud y de las ganas del profesor por querer hacer las cosas de la mejor manera (D.C., B.-2017).

\section{Las conclusiones sobre el propio trabajo en Prácticas}

Los datos muestran que el alumnado del Postgrado y Licenciatura analiza las acciones docentes desde el marco teórico genérico y no se observa una observación detallada de las tareas que se ejecutaban en clase, aunque pudiera parecer tal la frase del uso del libro de texto por el alumnado. Falta saber cómo lo utilizaba, cuáles eran los términos que no entendían, o cuáles las instrucciones desconocidas, o la falta de integración de las imágenes en la explicación discursiva.

Cuando se trataba de sintetizar su actuación en el Practicum hemos visto que se utilizaban parámetros diferentes a los del profesorado mentor/a, centrándose más en el comportamiento y en la relación humana emocional que en el análisis de los contenidos específicos para plantear y resolver problemas conceptuales, pese a que hay algunos ejemplos que nos manifiestan una impugnación de este sentido práctico de las actuaciones del profesorado, que a veces, como es el caso, está sostenido en una visión estereotipada del conocimiento del alumnado, como reconoce en sus anotaciones; así se identifican las concepciones espontáneas de los/las adolescentes para resolver sus problemas cotidianos. Sin embargo, la mayoría de las referencias aluden al control del comportamiento del alumnado y a la búsqueda de la satisfacción en la relación interpersonal.

He de decir que antes de las prácticas menospreciaba en cierta medida la capacidad que tenía el alumnado de secundaria, pero después de mi experiencia he visto que puedes introducir en ellos y ellas una visión crítica, tratar muchos temas y pedir cosas más complejas de lo que parece (E.S., V.-2020)

A su vez, he podido ir poniendo en práctica estrategias de control y de resolución de problemas, gestionando también habilidades de improvisación, algo que considero fundamental dentro de la docencia. (L.G., V.-2020)

Mi gran satisfacción en el Practicum fue cuando enseñé las clases y

\footnotetext{
${ }^{24}$ Original en catalán.
} 
escuché a los estudiantes que la clase era buena y que aprendieron el tema. (R.A.S., A.-2019)

Mis primeras clases fueron de observación de cómo se desarrollaba la clase, en esas primeras clases al ver lo productivo de la clase determiné que me cambiaría mi pensamiento... esto se discute en la universidad, pero con el profesor titular no se toca directamente, porque lo que interesa es cumplir con los programas establecidos (Y.C., B.2017).

Los ejemplos recogidos muestran las dificultades de aplicar las competencias institucionales (en el caso de UV son más de 20 competencias) en el momento de iniciar la carrera profesional, cuando existen implicaciones emocionales. Por eso queremos destacar frases como las siguientes:

J.B., B.-2019:Verdaderamente el rol del profesor más allá de enseñar, es aprender todos los dias y utilizar eso que aprende para su vida y para su profesión, no para transformar el mundo, pero si para transformarse a sí mismo (...) La relación entre teoría y práctica está justamente en la reflexividad docente, en que hay que poner su energía e intención en todas las actividades. Esto es desgastante y a veces frustrante porque las preparaciones de clase no siempre dan sus frutos. Te das cuenta de las diferentes presiones a las que está sometido el profesorado, como la falta de tiempo, exámenes de la PAU, presiones del AMPA o del resto del profesorado, necesidad de hacer cursos para obtener sexenios, etc. (E.S., V.-2020) 25.

Son frases que nos invitan a continuar investigando en la praxis escolar, pues nos muestran una gradación de categorías conceptuales para programar el desarrollo del Practicum.

\section{Referencias}

Bertazzo, C.J. (2019). Formação de professores de geografia e ciências sociais para o nível secundário em Espanha e Brasil, Praxis, Educación y Pedagogia, 3, 68-101. $10.25100 /$ praxis_educacion.v0i3.8321

Bisquerra, R. (2004). Metodología de la investigación educativa. Madrid: La Muralla.

Clark, Ch. y Peterson, P. (1990). Procesos de pensamiento de los docentes. En Witrock, M. (Comp). La investigación de la enseñanza. III. Barcelona: Paidós.

Coe, R. (2002). It's the Effect Size, Stupid. What effect size is and why it is important, Paper presented at the British Educational Research Association annual conference, Exeter, 1214.

${ }^{25}$ El texto original estaba en catalán. Las siglas PAU significan las Pruebas de Acceso a la Universidad y AMPA, las asociaciones de Madres y Padres de Alumnos. 
http://www.cem.org/attachments/ebe/ESguide.pdf]

Coiduras, J.L., Gervais, C. y Correa, E. (2009). El contexto escolar como escenario de educación superior en la formación de docentes. El Practicum en Quebec como modelo para reflexionar ante las nuevas titulaciones de grado. Educar 44, 11-29.

Domingos, M. (2019). Habitus docente, sens pratique e representações sociais. En Barbosa, M. F. (org.), Bourdieu e Moscovici: fronteiras, interfaces e aproximações (pp. 19-46). Editora Universitária Leopoldianum.

Domingos, M. y Diniz, A. A. (2019). Representações Sociais, Sens Pratique, Poder Simbólico e o Processo de Construção do Ser Docente. Arxius de Sociologia, 41, 43-64.

Escudero, J. M. (2009). La formación del profesorado de Educación Secundaria: contenidos y aprendizajes docentes. Revista de Educación, 350, 79-103.

Esteve, J.M (2006). La profesión docente en Europa: perfil, tendencias y problemática. La formación inicial. Revista de Educación, 340, p. 19-86.

Fernández, M. y Gurevich, R. (2018). Educación geográfica e investigación educativa en la formación de profesores. En García de la Vega, (ed). Reflexiones sobre educación geográfica. Revisión disciplinar e innovación educativa (pp. 27-44). Madrid: Universidad Autónoma de Madrid y Edições Lagim.

Fernández, M. (2001). A la busca de un modelo profesional para la docencia: ¿liberal, burocrático o democrático? Revista Iberoamericana de Educación. 25, 43-64.

González-Sanmamed, M. (2015). El practicum en la formación del profesorado de Secundaria. Revista Española de Pedagogía, $\mathrm{n}^{\circ}$ 261, p. 301-319.

Hernández, F. y Sancho, J. (1996). Para enseñar no basta con saber la asignatura. Barcelona: Editorial Paidós.

Jodelet, D. (1985): "La representación social, fenómenos, concepto, teorías", en S. Moscovici (Ed.), Psicología social II, pensamiento y vida social. Barcelona: Grupo Paidós; pp. 469-494.

Martínez, N. (2014). La Historia de España en los recuerdos escolares. Análisis, interpretación y poder de cambio de los testimonios de profesores y alumnos, Valencia. Nau Llibres.

Moner, C. y Oller, M. (2014). Las expectativas de los estudiantes ante el practicum del máster de formación del profesorado de la especialidad de Geografia e Historia. En Pages, J. y Santisteban, I. (eds). Una mirada al pasado y un proyecto de futuro y aprendizaje de las ciencias sociales (2) (pp.601-608). Barcelona: UAB-AUPDCS, 2014, 583-591.

Moscovici, S. (2011). A invenção da sociedade. Sociologia e Psicologia, Petropolis, Rio Janeiro: Editora Vozes Ltda 
Pérez, A. (1987). El pensamiento del profesor, vínculo entre la teoría y la práctica. Revista de Educación. 284, 199-222.

Perrenoud, P. (2004). Diez nuevas competencias para enseñar. Barcelona: Graó.

Prats, E., Marín-Blanco, A., y Álvarez-López, G. (2019). Elementos para el debate en la iniciación profesional docente en España. La iniciación profesional docente: marcos supranacionales y estudios comparados. En Valle, J.M. (coord) La iniciación profesional docente: Marcos supranacionales y estudios comparados (pp. 204220). Barcelona: Dykinson S.L. https://repositorio.uam.es/bitstream/handle/10486/688712/ele mentos_prats_2019.pdf?sequence $=1$

Rando, W. y R. Menges (1991). How practice is shaped by personal theories. New Directions for Teaching and Learning, 45, 7-14.

Rivero, P. y Souto, X.M. (2019), El máster de profesorado de educación secundaria. Una visión desde la especialidad de geografia e historia, Íber Didáctica de las Ciencias Sociales, Geografía e Historia. 97, 41-50.

Rodríguez, L.A. (2019). Territorios epistémicos y práctica pedagógica en las licenciaturas de biologia y ciencias sociales de la Universidad Distrital Francisco José de Caldas. (Tesis doctoral). Universidad Distrital Francisco José de Caldas. Bogotá.

Rodríguez, L.A. y Souto, X.M. (2019). El Practicum de Ciencias Sociales en dos Universidades Iberoamericanas. Educação \& Realidade, 24(44)4, 1-25. http://dx.doi.org/10.1590/2175-623688073

Sánchez, I. (1996). Metodología de la investigación educativa y desarrollo de la profesión docente (Referencia a la educación secundaria). Revista Complutense de Educación, 7(2), 107-133. https://revistas.ucm.es/index.php/RCED/article/view/RCED969 6220107A

Santos, M. F. P (2012). O Estágio enquanto espaço de Pesquisa: caminhos a percorrer na formação docente em Geografia. (Tesis inédita de Doctorado). Universidade Federal do Rio Grande do Sul, Porto Alegre.

Santos, M. F. P. y Souto, X.M. (2018). A Educação Geográfica em Construção. Revista Terra Livre, 31(1), 46, 79-113.

Santos, M.F. P. (2019). El Practicum en Geografia: un locus para problematizar la formación inicial docente. En Parra, D. y Fuertes, C. (eds). Reinterpretar la tradición, transformar las prácticas: Ciencias Sociales para una educación critica (pp.358.372). Valencia: Tirant lo Blanch.

Schön, D.A. (1992). La formación de profesionales reflexivos: Hacia el nuevo diseño de enseñanza y aprendizaje. Barcelona: MEC, Paidós. Solbes, J., Fernández, J., Domínguez, M.C; Cantó, J., y Guisasola, J. (2018). Influencia de la Formación y la Investigación Didáctica del 
Profesorado de Ciencias sobre su Práctica Docente. Enseñanza de las Ciencias. 36(1), 25-44.

Souto, X. M. (2018). ¿Es posible innovar la praxis escolar desde el Practicum? Un estudio de caso en la Universitat de València. En López, E., García, C. y Sánchez, M. (eds). Buscando formas de enseñar: Investigar para innovar en didáctica de las ciencias sociales (pp. 275-286). Valladolid: Ediciones de la Universidad de Valladolid y AUPDCS. 
